\title{
Details of out-field regional recurrence after involved-field irradiation with concurrent chemotherapy for locally advanced esophageal squamous cell carcinoma
}

\author{
This article was published in the following Dove Press journal: \\ OncoTargets and Therapy \\ 23 May 2016 \\ Number of times this article has been viewed
}

\author{
Xiaoli Zhang ${ }^{1,2}$ \\ Jinming $Y u^{1,2}$ \\ Minghuan $\mathrm{Li}^{2}$ \\ Hui Zhu \\ 'Department of Oncology, Renmin \\ Hospital of Wuhan University, Wuhan, \\ ${ }^{2}$ Department of Radiation Oncology, \\ Shandong Cancer Hospital affiliated \\ to Shandong University, Shandong \\ Academy of Medical Sciences, Jinan, \\ People's Republic of China
}

\begin{abstract}
Background: The purpose of this study was to describe the patterns of out-field regional recurrence after involved-field irradiation (IFI) in definitive concurrent chemoradiotherapy (CCRT) for locally advanced esophageal squamous cell carcinoma (LA-ESCC) and identify the possible risk factors.

Patients and methods: Eighty patients with LA-ESCC who received CCRT with IFI between January 2003 and January 2009 at the Shandong Cancer Hospital were recruited and analyzed. Imaging scans demonstrating first sites of failure were compared with original computed tomography-based radiation treatment plans, and failure patterns were defined as in-field, outfield regional (failures in initially uninvolved regional nodes), and distant failures.

Results: After a median follow-up time of 52.6 months, 24 patients had evidence of out-field regional failure, 43 patients had evidence of in-field failure, and 33 patients had the evidence of distant failure. Multivariate analysis revealed that out-field regional failure was associated with clinical tumor status (T4 vs T1-3, odds ratio [OR] $=6.547, P=0.002)$, tumor length $(>8 \mathrm{~cm}$ vs $\leq 8 \mathrm{~cm}, \mathrm{OR}=4.130, P=0.036$ ), response to CCRT (complete response vs no complete response, $\mathrm{OR}=2.646, P=0.035$ ), and in-field failure (no in-field failure vs in-field failure, $\mathrm{OR}=1.32$, $P=0.016$ ). Survival analyses indicated that, compared to in-field failure or distant failure alone group, out-field regional failure alone group tended to have longer overall $(P=0.006)$ and progression-free survival $(P=0.164)$.

Conclusion: Our data suggested that the predominant failure pattern after IFI was not out-field regional failure, which also did not influence survival significantly, and that out-field regional failure did not shorten the time to disease recurrence, which also did not influence survival significantly. In addition, out-field regional failure was likely to appear later than in-field and distant failures. The relatively advanced local disease followed by poor local control and distant metastases contributed more to the poor outcome of LA-ESCC. Further prospective studies are needed to verify the findings of this study.
\end{abstract}

Keywords: failure patterns, prognosis, risk factors

\section{Introduction}

Esophageal cancer, as a highly invasive and metastatic tumor, ranks among the top five leading causes of morbidity and mortality in the People's Republic of China. ${ }^{1}$ Due to its biological and histological specificity, lymph node (LN) metastases are common, appearing early in the disease and often in "skip" patterns. ${ }^{2-4}$ A large majority of esophageal cancers are diagnosed at locally advanced stage with extensive nodal 
spread. Based on the results of Radiation Therapy Oncology Group 8501, concurrent chemoradiotherapy (CCRT) has been broadly applied as a standard management for patients with locally advanced esophageal squamous cell carcinoma (LA-ESCC). ${ }^{5}$ However, the radiation fields to be used in the treatment of LA-ESCC are controversial.

Recent reports as well as our previous research have reported that using three-dimensional conformal radiotherapy (3D-CRT) without intentional elective node irradiation (ENI) led to a rate of isolated out-field failure of only $2 \%-13 \%{ }^{6-10}$ Hence, some researchers thought that the paucity of elective nodal failures (ie, recurrence in a nodal region without preexisting gross disease) may be in part due to the large competing risk of local recurrence, thereby obviating any regional control benefit from ENI. In the definitive chemotherapy setting, however, improved local control may uncover a benefit for aggressive prophylactic nodal irradiation. Thus, the omission of ENI did not sacrifice the overall survival (OS); to some extent that suggested the feasibility of in-field irradiation (IFI; nodal target volume included only the malignant nodes) using definitive 3D-CRT for locally advanced cases.

However, the trend toward treating LA-ESCC with involved-field irradiation (IFI) has generated concern for the increased risk of nodal failure in untreated nodal area, as clinically uninvolved LNs may harbor microscopic disease. ${ }^{6-10}$ The purpose of this study was to retrospectively describe the patterns of out-field recurrence of IFI in CCRT for LA-ESCC and then identify the clinical factors that may be associated with failure in out-field volume.

\section{Patients and methods}

\section{Patients}

Use of IFI with concurrent chemotherapy for esophageal cancer has been routine in the Department of Radiation Oncology (Shandong Cancer Hospital affiliated to Shandong University) since 2003. We retrospectively reviewed the clinical records of patients with LA-ESCC, who had been histologically confirmed by biopsy or brush sample between January 2003 and December 2009. All the included patients had no malignant tumor history and had received definitive IFI with concurrent chemotherapy consisting of cisplatin and fluorouracil. Patients underwent a series of standardized evaluations, including esophagography, endoscopic ultrasonography, and computed tomography (CT), and were eventually staged according to the American Joint Committee on Cancer and International Union Against Cancer TNM staging system (Version 7.0, 2009). ${ }^{11}$ Some patients also underwent position emission tomography (PET) as part of $\mathrm{PET} / \mathrm{CT}$ fusion scans. This retrospective study was approved by the institutional review board of Shandong Cancer Hospital. All patients included in this study have voluntarily provided written informed consent.

\section{Chemoradiotherapy}

Details of chemoradiotherapy have been published previously. ${ }^{9}$ All the patients had received definitive radiotherapy with concurrent chemotherapy consisting of two cycles of cisplatin $\left(75 \mathrm{mg} / \mathrm{m}^{2} / \mathrm{d}\right.$ on day 1) and fluorouracil $\left(700 \mathrm{mg} / \mathrm{m}^{2} / \mathrm{d}\right.$ as a continuous infusion on days $\left.1-4\right)$ every 21 days. An additional one or two (median 2) cycles of chemotherapy with the same regimen were given to 57 patients (71\%) once treatment with CCRT was over.

The gross tumor volume (GTV) was contoured based on all available resources (esophagography, endoscopic ultrasonography, and diagnostic CT and PET/CT fusion scans). The GTV included the GTV of esophageal tumor (GTVe) and GTV of clinically involved nodes (GTVn). The criteria for metastatic LNs included well vasculated, measuring $>1.0 \mathrm{~cm}$ in the short axis or $>1.5 \mathrm{~cm}$ in the long axis on CT scans, or nodes with high uptake of fluorodeoxyglucose on PET images (maximum standard uptake value $\geq 2.5$ ). The GTVe was expanded to the clinical target volume (CTV) of the esophagus by extending the radiation coverage to $3-4 \mathrm{~cm}$ superiorly, $3-4 \mathrm{~cm}$ inferiorly, and $0.8-1.0 \mathrm{~cm}$ laterally. A margin of $0.5-1.0 \mathrm{~cm}$ to cover the microscopic spread of disease was added to GTVn to form the CTV of clinically involved nodes GTVn. The planning target volume (PTV) was then generated using a uniform 0.5-1.0 cm expansion beyond the borders of the CTV. Considering that the radiation dose of $50.4 \mathrm{~Gy} / 28$ fractions specified in Radiation Therapy Oncology Group 8501 was inadequate for local control, all patients in our study were treated with a total dose of 50-64 Gy given in 25-32 fractions (median dose 60 Gy). Radiation treatments in 58 of the 80 patients were delivered as 3D-CRT, while in 22 of the 80 patients, the treatments were delivered as intensity-modulated radiation therapy with standard fractionation (2.0 Gy fractions given daily for 5 days/week); treatment plans were generated with a 3D planning system (ADAC-Pinnacle 3, Version 5.0; Philips Radiation Oncology Systems, Fitchburg, WI, USA).

\section{Result assessment and follow-up}

Routine examinations (enhancement CT scans, ultrasonography, PET/CT, esophagography, endoscopic ultrasonography, as well as repeat blood work) were performed for all the patients included, 1 month after the completion of treatment, every 3 months for 2 years, and once yearly thereafter. For the total group of 80 patients, Response Evaluation Criteria in Solid Tumors system was used to evaluate the treatment 
response as well as failure patterns. ${ }^{12}$ Briefly, responses were classified as complete response (CR), partial response, progressive disease, or stable disease. Patterns of failure were assessed based on the result of posttreatment esophagography, endoscopic ultrasonography, CT, or PET/CT scans and compared with the radiation treatment plans which were made on the basis of the original enhanced CT image. Failure models were defined according to the first sites of failure as in-field, out-field regional LN, and distant failures. In-field recurrences included the primary lesion and involved nodes. Out-field regional LN failures were failures in initially uninvolved regional nodes defined as the periesophageal nodes ranging from the cervical nodes to the celiac nodes. ${ }^{13}$ Nodal metastases outside the region were defined as distant failure. Suspected esophageal recurrences were confirmed by histological or cytologic testing. Nodes that reappeared after having completely disappeared or became larger after having remained stable, and new nodes that appeared in the mediastinal or abdominal regions where no enlarged nodes had been seen before irradiation were considered nodes' recurrences. When supraclavicular node recurrence was suspected, it was confirmed by needle aspiration. Survival intervals (OS and progression-free survival [PFS]) were calculated from the first day of CCRT.

\section{Statistical analysis}

Continuous variables were summarized by descriptive statistics, such as medians and standard deviations. Categorical variables were tabulated by frequency and percentage. Fisher's exact test or chi-square test and Wilcoxon rank sum test were used to compare patient characteristics between patients with and without out-field regional failure. Logistic regression models were fit for multivariate analysis to evaluate the associations between out-field regional failure and any of the clinical factors with $P<0.05$ on univariate analysis. The Kaplan-Meier method and log-rank tests were applied to estimate the survival probabilities and compare survival between groups. OS and PFS were calculated using the Kaplan-Meier method from the date of radiation therapy treatment initiation. The Statistical Package for the Social Sciences Version 17.0 (SPSS Inc., Chicago, IL, USA) software was used for data analysis.

\section{Results \\ Patients, disease, and treatment characteristics}

Table 1 shows the clinical and treatment characteristics of the 80 patients included in this retrospective analysis. In addition, the median patient age was 63 years (range, 42-74 years), and the median tumor length was $5 \mathrm{~cm}$ (range, 2-13 cm). PET was included in the staging procedures for eleven patients.

\section{Patterns of first failure}

Analysis of recurrence data was made at the time of last follow-up contact in December 2011 (median follow-up interval of 52.6 months [95\% CI, 46.1-56.7 months]); failure

Table I Patient characteristics and Fisher's exact test or chi-square test to determine the association of out-field regional LN failure and covariates

\begin{tabular}{|c|c|c|c|c|}
\hline \multirow[t]{2}{*}{ Variable } & \multirow[t]{2}{*}{ Entire cohort (\%) } & \multicolumn{2}{|c|}{ Failure in out-field regional LN } & \multirow[t]{2}{*}{$P$-value } \\
\hline & & No (\%) & Yes (\%) & \\
\hline Sex & & & & 0.759 \\
\hline Male & $52(65.0)$ & 37 (7I.2) & $15(28.8)$ & \\
\hline Female & $28(35.0)$ & $19(67.9)$ & $9(32.1)$ & \\
\hline Age (years) & & & & 0.450 \\
\hline$\leq 60$ & $30(37.5)$ & $23(76.7)$ & $7(23.3)$ & \\
\hline$>60$ & $50(62.5)$ & $33(66.0)$ & $17(34.0)$ & \\
\hline Clinical T stage & & & & $<0.000 I^{*}$ \\
\hline $\mathrm{TI}-3$ & $63(78.8)$ & $53(84.1)$ & $10(15.9)$ & \\
\hline $\mathrm{T} 4$ & $17(2 \mid .3)$ & $3(17.6)$ & $14(82.4)$ & \\
\hline Clinical $N$ stage & & & & 0.056 \\
\hline No & $21(26.3)$ & I8 (85.7) & $3(14.3)$ & \\
\hline $\mathrm{N}+$ & $59(73.8)$ & $38(64.4)$ & $21(35.6)$ & \\
\hline Primary tumor location & & & & 0.648 \\
\hline Upper thoracic & $21(26.3)$ & $14(66.7)$ & $7(33.3)$ & \\
\hline Middle thoracic & $49(61.3)$ & $36(73.5)$ & $13(26.5)$ & \\
\hline Lower thoracic & $10(12.5)$ & $6(60.0)$ & $4(40.0)$ & \\
\hline Tumor length $(\mathrm{cm})$ & & & & $0.027^{*}$ \\
\hline$\leq 8$ & 75 (93.8) & $55(73.3)$ & $20(26.7)$ & \\
\hline$>8$ & $5(6.3)$ & I (20.0) & $4(80.0)$ & \\
\hline
\end{tabular}


Table I (Continued)

\begin{tabular}{|c|c|c|c|c|}
\hline \multirow[t]{2}{*}{ Variable } & \multirow[t]{2}{*}{ Entire cohort (\%) } & \multicolumn{2}{|c|}{ Failure in out-field regional LN } & \multirow[t]{2}{*}{$P$-value } \\
\hline & & No (\%) & Yes (\%) & \\
\hline Receiving PET scans at diagnosis & & & & 0.291 \\
\hline Yes & II (I3.8) & $6(54.5)$ & $5(45.5)$ & \\
\hline No & $69(86.3)$ & $50(72.5)$ & $19(27.5)$ & \\
\hline Radiation dose (Gy) & & & & 0.078 \\
\hline$<60$ & II (I3.8) & $5(45.5)$ & $6(54.5)$ & \\
\hline$\geq 60$ & $69(86.3)$ & $51(73.9)$ & $18(26.1)$ & \\
\hline Radiation technique & & & & 0.183 \\
\hline 3D-CRT & $58(72.5)$ & $38(65.5)$ & $20(34.5)$ & \\
\hline IMRT & $22(27.5)$ & I8 (8I.8) & $4(18.2)$ & \\
\hline Adjuvant chemotherapy & & & & $0.002^{*}$ \\
\hline Yes & $57(71.3)$ & $46(80.7)$ & II (19.3) & \\
\hline No & $23(28.8)$ & $10(43.5)$ & $13(56.5)$ & \\
\hline Pathological complete response & & & & $0.045^{*}$ \\
\hline Yes & $19(23.8)$ & $17(89.5)$ & $2(10.5)$ & \\
\hline No & $61(76.3)$ & 39 (63.9) & $22(36.1)$ & \\
\hline In-field failure & & & & $<0.000 I^{*}$ \\
\hline Yes & $43(53.8)$ & $39(90.7)$ & $4(9.3)$ & \\
\hline No & $37(46.3)$ & 17 (45.9) & $20(54.1)$ & \\
\hline Distant failure & & & & 0.143 \\
\hline Yes & $33(41.3)$ & $20(60.6)$ & $13(39.4)$ & \\
\hline No & $47(58.8)$ & $36(76.6)$ & II (23.4) & \\
\hline
\end{tabular}

Note: *Statistically significant.

Abbreviations: 3D-CRT, three-dimensional conformal radiotherapy; IMRT, intensity-modulated radiation therapy; LN, lymph node; PET, positron emission tomography.

had occurred in 76 (95\%) patients: $43(53.8 \%)$ patients in-field, 24 (30\%) patients out-field regional nodes, and 33 (41.3\%) patients distant. Among them, 21 (26.3\%) patients had failed at more than one site: one patient in both in-field and out-field regional volumes, seven patients in both in-field and distant volumes, ten patients in both out-field regional and distant volumes, and three patients in in-field, out-field regional, and distant volumes. In-field, out-field regional node, and distant failures as the only sites of failure were seen in 32 (40\%), 10 (12.5\%), and 13 (16.25\%) patients, respectively.

\section{Location of out-field regional LN failure}

Of the 24 patients with out-field regional failure, 14 patients also had failures at other sites: one patient with in-field failure, ten patients with distant failure, and three patients with both in-field and distant failures. Location of out-field regional nodes are shown in Figure 1. There were a total of 55 recurrences in these 24 patients; these were located in left (L)- (five patients) and right (R)-supraclavicular (three patients), L- (six patients) and R-upper mediastinum (eight patients), prevascular nodes and retrotracheal nodes (one patient), L- (five patients) and R-lower paratracheal nodes (seven patients), aortic-pulmonary window (three patients), para-aortic nodes (one patient), subcarinal nodes (three patients), paraesophageal nodes (one patient), L- (one
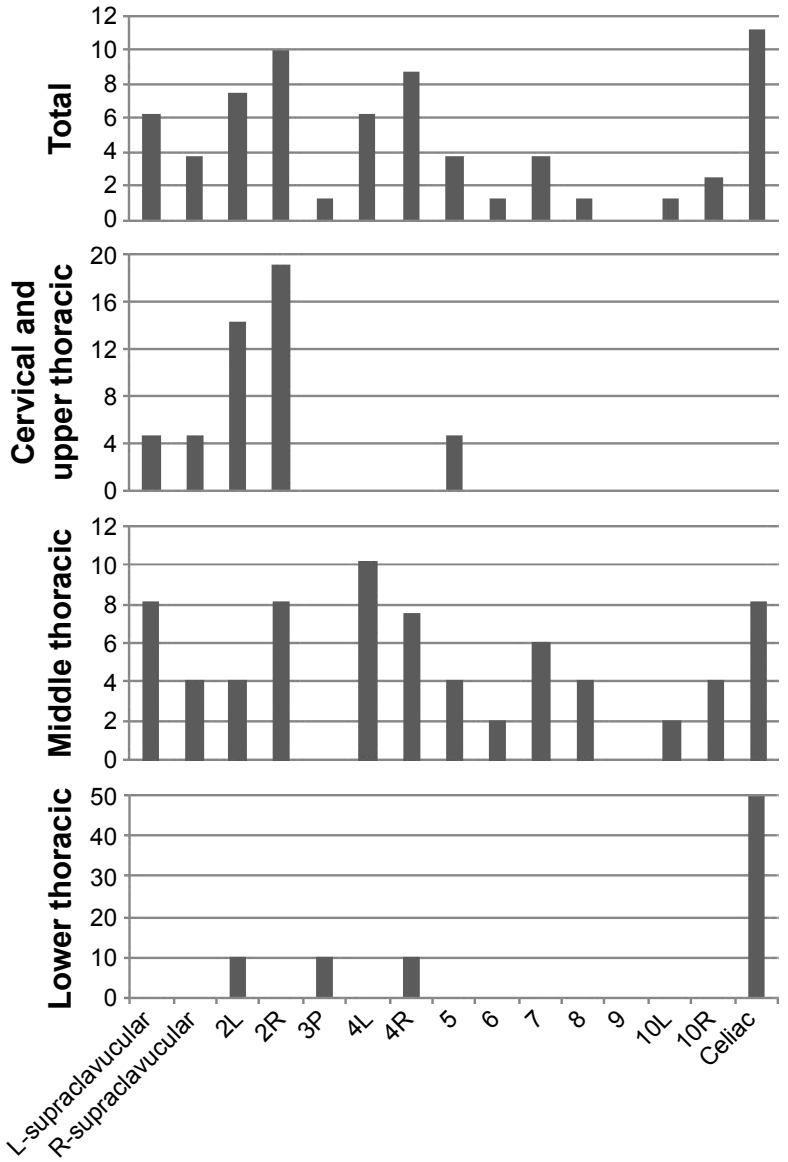

Figure I Rate of out-field regional lymph node failure in different sites. Abbreviations: L, left; $R$, right. 
patient) and R-hilar nodes (two patients), and celiac (nine patients).

\section{Predictors of out-field regional failure}

To identify the clinical factors, if any, that may be associated with failure in the out-field regional LN, we used Fisher's exact tests and Wilcoxon rank sum test to compare the variables for patients with and without out-field regional failure. On univariate analysis, out-field regional failure was associated with clinical T stage $(P<0.0001)$, tumor length ( $P=0.027)$, not receiving adjuvant chemotherapy $(P=0.002)$, no CR to CCRT $(P=0.045)$, and in-field failure $(P<0.0001)$. Tumor stage was strongly associated with out-field regional control; 14 of the $24(58.3 \%)$ patients with T4 disease had out-field regional failure vs only ten of the $24(41.7 \%)$ patients with T1-3 disease $(P<0.0001)$. In addition, the univariate analysis also suggested that receiving adjuvant chemotherapy could also decrease the risk of developing out-field regional failure $(P=0.002)$. Patients with regional $\mathrm{LN}$ involved before treatment had a trend of higher risk of experiencing out-field regional failure $(P=0.056)$. Interestingly, the rate of failure within out-field regional volume was found to be higher among patients without in-field failure (20/37 [54.1\%] vs $4 / 43$ [9.3\%]) than those who experienced in-field failure $(P<0.0001)$ (Table 1).

On multivariate analysis, having a T4 tumor compared to a T1-3 tumor was associated with a higher risk of outfield regional failure (odds ratio [OR], 6.547; 95\% CI, $1.823-21.857 ; P=0.002$ ). Risk of out-field regional failure was associated with no pathological CR after chemoradiation, with patients of no CR conferring a higher risk of experiencing out-field regional failure (OR, 2.646; 95\% CI, $1.374-4.975 ; P=0.035)$. Tumor length as a category variable $(>8 \mathrm{~cm})$ was a predictor of out-field regional failure on multivariate analysis (OR, 4.130; 95\% CI, 1.454-10.960; $P=0.036$ ). Consistent with the univariate analysis, the multivariate analysis also suggested that patients with in-field failure were associated with a lower risk of developing out-field regional failure (OR, 1.32; 95\% CI, 1.101-1.456; $P=0.016$ ) (Table 2).

\section{Response and survival}

As reported in our last article, the overall response was $85 \%$ (24\% CR and 61\% partial response). The median PFS time was 11.3 months (95\% CI, 8.8-13.2 months), and the 1-, 2-, and 3-year PFS rates were 41.3, 18.9, and $11.3 \%$, respectively (Figure 2A)..$^{9}$ The median OS time was 14.4 months (95\% CI, 13.4-15.9 months), and the 1-, 2-,
Table 2 Multivariate logistic regression analysis for out-field regional LN failure

\begin{tabular}{|c|c|c|}
\hline Variable & OR $(95 \% \mathrm{Cl})$ & $P$-value \\
\hline \multicolumn{3}{|c|}{ Clinical T stage } \\
\hline \multicolumn{3}{|l|}{$\mathrm{TI}-3$} \\
\hline $\mathrm{T} 4$ & $6.547(|.823-2| .857)$ & 0.002 \\
\hline \multicolumn{3}{|c|}{ Pathological complete response } \\
\hline \multicolumn{3}{|l|}{ Yes } \\
\hline No & $2.646(1.374-4.975)$ & 0.035 \\
\hline \multicolumn{3}{|c|}{ Length $(\mathrm{cm})$} \\
\hline \multicolumn{3}{|l|}{$\leq 8$} \\
\hline$>8$ & $4.130(1.454-10.960)$ & 0.036 \\
\hline \multicolumn{3}{|c|}{ In-field failure } \\
\hline \multicolumn{3}{|l|}{ Yes } \\
\hline No & $1.320(1.101-1.456)$ & 0.016 \\
\hline
\end{tabular}

Abbreviations: LN, lymph node; OR, odds ratio.

and 3-year OS rates were $86.3,30.0$, and 18.8 , respectively (Figure 2B).

According to the different failure patterns, the median PFS times for the in-field recurrence arm, out-field regional recurrence arm, and distant failure arm were 8.9, 13.1, and 12.0 months, respectively $(P=0.219$, Figure $3 \mathrm{~A})$. The median survival times for the corresponding arms were 14.2, 14.5, and 13.2 months, respectively ( $P=0.189$, Figure 3B). No significant difference was found in the in-field recurrence $\operatorname{arm}(P=0.258)$, out-field regional recurrence $\operatorname{arm}(P=0.279)$, as well as the distant failure arm $(P=0.508)$ after comparing the PFS of different failure patterns with the overall PFS. Comparison between the OS of different failure patterns and the overall OS showed a worse survival for patients with in-field $(P=0.037)$ or distant $(P=0.004)$ failure, but not for patients with out-field failure $(P=0.061)$, respectively. For patients with isolated failure patterns, the PFS times of infield, out-field regional, and distant failures alone were 10.1, 13.8, and 11.2 months, respectively ( $P=0.164$, Figure $4 \mathrm{~A})$. The median OS times of isolated in-field, out-field regional, and distant failure groups were 24.5, 35.3, and 18.2 months, respectively ( $P=0.006$, Figure 4B). Comparison between the PFS of different isolated failure patterns and the overall PFS revealed no significant difference (in-field recurrence arm, $P=0.258$; out-field regional recurrence arm, $P=0.279$; distant failure arm, $P=0.508)$. The $\mathrm{OS}$ of patients with isolated infield $(P=0.016)$ or out-field regional $(P=0.028)$ failure, but not distant failure $(P=0.800)$, was significantly better than the overall OS.

\section{Salvage treatment}

During the follow-up, 65 patients received salvage therapy after treatment failure. Among them, 32 patients received 

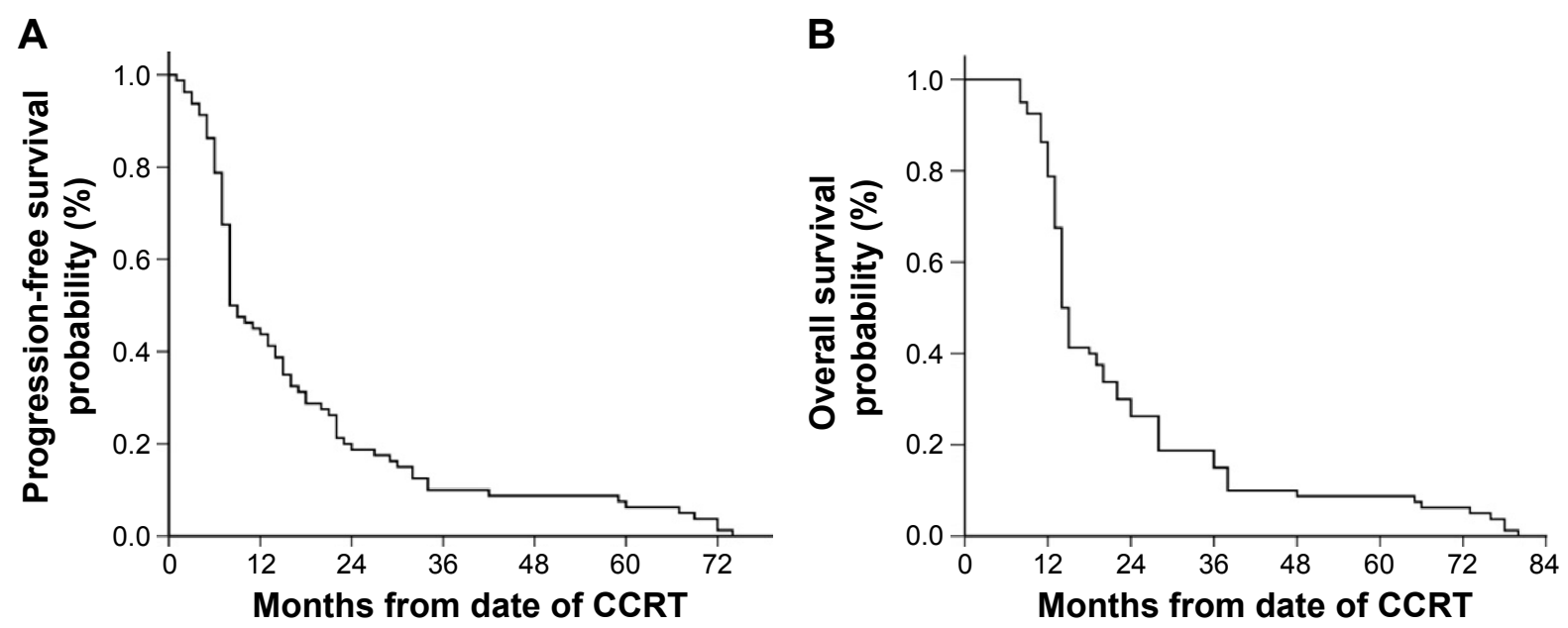

Figure 2 Kaplan-Meier progression-free survival curves $(\mathbf{A})$ and overall survival rates curves (B) for patients with LA-ESCC treated with chemoradiation with involved-field irradiation.

Abbreviations: CCRT, concurrent chemoradiotherapy; LA-ESCC, locally advanced esophageal squamous cell carcinoma.

chemotherapy, 35 patients received palliative radiation, and eight patients received surgery. All the 10 patients with solitary regional $\mathrm{LN}$ failure received salvage treatment; among them, four patients experienced $>24$ months survival period after the salvage treatment.

\section{Discussion}

In our previous analysis of the failure patterns and survival of patients with LA-ESCC receiving IFI with concurrent chemotherapy, we found that the vast majority of recurrences occurred within in-field or distant volume. ${ }^{9}$ In addition, despite the relatively high risk of out-field regional failure $(24 / 80)$ in our series, most patients experienced concurrent in-field failure or distant metastases, implying that the disease was insensitive to both local-regional and systemic therapies, and additional regional treatment may not improve the disease course. Other reports of patients with esophageal cancer treated with radical CCRT have yielded similar patterns of failure..$^{6-8,10} \mathrm{~A}$ recent study that retrospectively compared the failure patterns with the effects of ENI or IFI on patients with cervical and upper thoracic ESCC had reported that isolated "out-PTVifi in-PTVeni" cervical node metastasis occurred in three patients (3\%) in the IFI group and two patients (3\%) in the ENI group, and
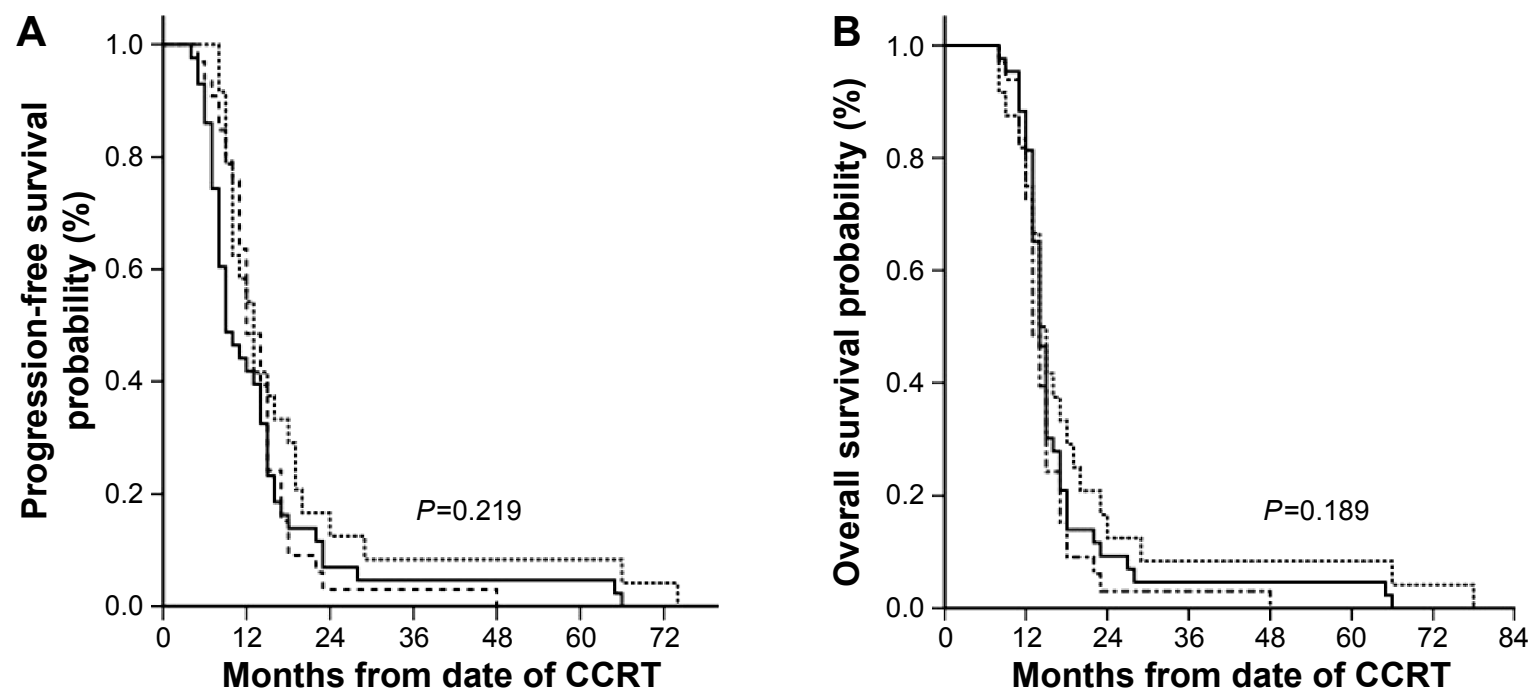

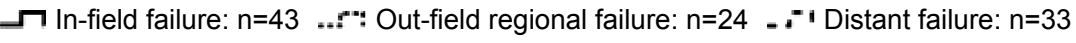

Figure 3 Kaplan-Meier estimates of progression-free survival (A) and overall survival (B) for patients with LA-ESCC with different failure patterns. Abbreviations: CCRT, concurrent chemoradiotherapy; LA-ESCC, locally advanced esophageal squamous cell carcinoma. 

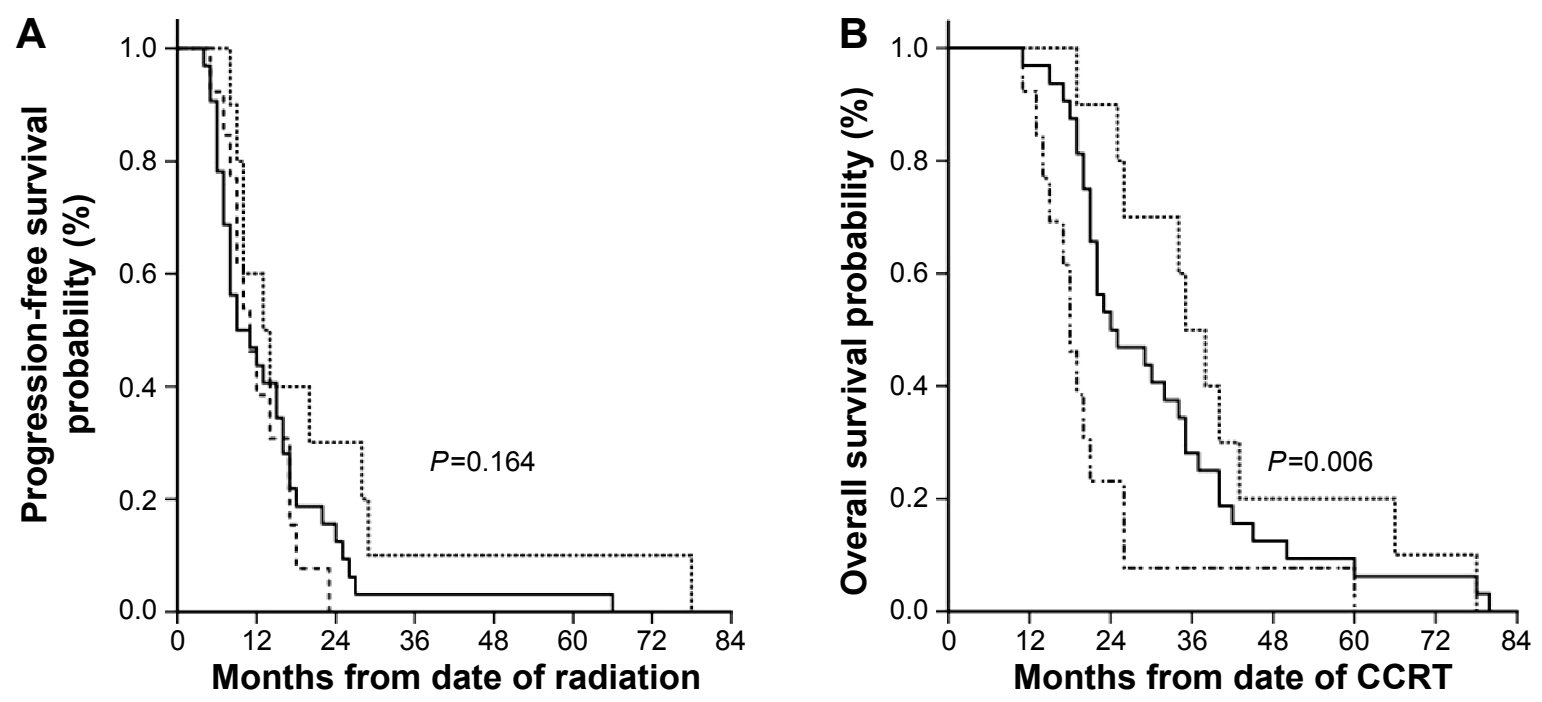

In-field failure: $n=32 \ldots$

Figure 4 Kaplan-Meier estimates of progression-free survival $(\mathbf{A})$ and overall survival (B) for patients with LA-ESCC with different isolated failure patterns. Abbreviations: CCRT, concurrent chemoradiotherapy; LA-ESCC, locally advanced esophageal squamous cell carcinoma.

the 3-year OS rates for the IFI and the ENI group were $49 \%$ and $47 \%$, respectively $(P=0.741) .{ }^{10}$ Analysis of survival in our previous research also revealed that the OS for patients with and without out-field regional failure showed no statistically significant difference. Thus, we think that prophylactic nodal treatment in these patients would likely not have an improved disease control due to the competing risks of infield and distant failures.

In this study, we attempted to retrospectively describe out-field regional failure patterns of IFI in CCRT for LAESCC in detail and then identify the clinical factors, if any, that may be associated with failure in out-field region. We identified several pretreatment factors as being associated with the risk of out-field regional failure, including having a T4 tumor and a tumor $>8 \mathrm{~cm}$ in size. Tumors of T4 stage or having a longer size $(>8 \mathrm{~cm})$ at diagnosis with out-field regional failure rates of $14 / 17(82.4 \%)$ and $4 / 5(80 \%)$ vs only $10 / 63(15.9 \%)$ and 20/75 (26.7\%) for tumors of T1-3 stage or having a shorter size $(\leq 8 \mathrm{~cm})$ suggested that advanced stage or longer tumors may have a higher risk of developing out-field regional failure after definitive IFI with concurrent chemotherapy. Previous studies have also reported that advanced stage of tumor at diagnosis, especially T4, was a negative factor for local-regional control. ${ }^{7,14-16}$ In addition, we also identified several posttreatment risk factors as being associated with the risk of out-field regional failure. Our data suggested that patients without CR to treatment were more likely to develop out-field regional failure. Yamashita et $\mathrm{al}^{17}$ using extended field reported that, among the 87 patients
(69\%) who achieved CR without any residual tumor after completion of chemoradiotherapy, local progression and distant metastases occurred in 20 (16\%) and 20 (16\%) patients, respectively, with or without other failure patterns, while isolated nodal failure was not found in any patient. Onozawa et $\mathrm{al}^{18}$ reported that after achieving $\mathrm{CR}$, only one patient experienced elective nodal failure without any other site of recurrence. Their results reflect our own. Thus, whether in ENI or IFI group, the response of tumor to treatment plays an important role in predicting regional recurrence. Consistent with this hypothesis was the finding in previous studies that only patients with histological CR could acquire survival benefits. ${ }^{19-21}$

Statistical analysis of survival times showed no significant difference in median OS and DFS among different failure groups. However, for patients with solitary failure patterns, the longest median survival time was found in the group of out-field regional failure alone. Although no statistically significant difference was found in median DFS among different failure groups, the DFS time of out-field failure group tended to be longer than in-field and distant failure groups. Therefore, we think the relatively low rate of outfield regional nodal recurrence is, thus, likely the result of the high incidence of esophageal failures and distant metastases, which may have masked regional nodal disease because many of the patients died before their regional nodal failure became clinically apparent.

In this study, the prevalence of LN metastasis varied in esophageal cancer of different locations. We speculated that 
out-field regional LN metastases (LNM) in upper thoracic ESCC tended to appear upward, whereas out-field regional LNM in lower thoracic ESCC tended to appear downward. In middle thoracic ESCC, out-field regional LNM could appear both upward and downward. If those sites with an out-field regional LNM rate of $>15 \%$, an empirical cut-off value, were considered as high-risk areas and should be included in the target volume, only $2 \mathrm{~L}$, celiac regional LN should be included in the target volume of cervical/upper thoracic and lower thoracic ESCC. Skip metastases were also observed in ESCC of all sites, which was consistent with previous reports. ${ }^{2-4}$ Hosch et $\mathrm{al}^{2}$ demonstrated that skip metastases were frequently found in esophageal cancer through both histopathological and immunohistochemical detections. With respect to the complex lymphatic drainage of esophageal cancer, the esophagus has an extensive longitudinal interconnecting system of lymphatics that enables lymph to travel the entire length of the esophagus before draining into LNs. ${ }^{22}$ Therefore, it is difficult to define in advance the accurate margin of prophylactic out-field regional LN due to the fact that ESCC is greatly apt to metastasis with a vague, extensive, and unpredicted invasion range.

As shown in our previous study, a large majority of patients experienced recurrences in more than one site, and out-field regional failure was only one of all the treatment failure modes. Therefore, ENI was most likely not a solution in these patients. Only a systematic adjuvant therapy (radiotherapy, chemotherapy, surgery, immunotherapy, etc) could bring survival benefit.

When discussing radiation fields, acute and late toxicities should also be considered. For patients with locally advanced stage, the target volume was sometimes large, due to the relatively long local lesion or serious esophageal invasion or extensive clinical node metastases. When used for the treatment of ESCC, IFI was observed to reduce the amount of normal lung parenchyma, cardiac, and esophagus that were irradiated. Kersting et al assessed late toxicities associated with IFI and reported that $<5 \%$ patients experienced grade 3 or greater late toxicities. ${ }^{19}$ Even though ENI may reduce the local/regional failures, substantial side effects may offset its survival benefits. ${ }^{18}$ In a study conducted by Ishikura et al, ${ }^{23}$ of 78 patients who achieved CR after CRT with ENI, 16 patients suffered from late cardiopulmonary toxicities and eight patients were believed to have died from toxicities related to CRT. Thus, IFI may result in a reduced incidence of treatment toxicities that enable more patients to tolerate the CCRT.
Among the limitations of this study were its retrospective nature, with the associated biases, and the fact that more advanced techniques for clinical staging of disease have come into service over the 6-year time span of the study. Endoscopic ultrasonography, PET scanning, and other functional imaging techniques were not available for all patients, which might have led to diagnostic underestimation and influenced the target volume for IFI. Though the statistical analysis found no significant difference in out-field regional failure rates between patients using or not using PET/CT for pretreatment evaluation in our study, the relatively small number of patients receiving $\mathrm{PET} / \mathrm{CT}$ might have affected the results. Other factors also weaken the strengths of our analysis, including the relatively rare form of esophageal cancer (most of the patients included had middle or lower esophageal cancer), the consistency of the treatment dose, and technique used over the relatively long period of observation.

\section{Conclusion}

To sum up, we found that out-field regional failure was not the predominant failure pattern after definitive chemoradiation with IFI for LA-ESCC. Out-field regional failure tended to happen later compared with in-field or distant failure, which may have led to less effect on survival time. Thus, we think additional ENI might be unnecessary and IFI might be feasible for patients with LA-ESCC. However, further investigations with large sample size, multicenter, prospective, stratified, randomized clinical trials are in need to verify our current conclusion.

\section{Acknowledgment}

This work was supported by the Natural Science Foundation of Shandong Province (ZR2012L35), People's Republic of China. The abstract of this paper was presented at the Annual Meeting Program Committee of the American Society for Radiation Oncology (ASTRO) as a poster presentation.

\section{Disclosure}

The authors report no conflicts of interest in this work.

\section{References}

1. Chen W, He Y, Zheng R, et al. Esophageal cancer incidence and mortality in China, 2009. J Thorac Dis. 2013;5:19-26.

2. Hosch SB, Stoecklein NH, Pichlmeier U, et al. Esophageal cancer: the mode of lymphatic tumor cell spread and its prognostic significance. J Clin Oncol. 2001;19:1970-1975.

3. Chen J, Liu S, Pan J,et al. The pattern and prevalence of lymphatic spread in thoracic oesophageal squamous cell carcinoma. Eur J Cardiothorac Surg. 2009;36:480-486. 
4. Nomura T, Onda M, Miyashita M, et al. Wide-spread distribution of sentinel lymph nodes in esophageal cancer. J Nippon Med Sch. 2001;68: 393-396.

5. Cooper JS, Guo MD, Herskovic A, et al. Chemoradiotherapy of locally advanced esophageal cancer: long-term follow-up of a prospective randomized trial (RTOG 85-01). Radiation Therapy Oncology Group. JAMA. 1999;281:1623-1627.

6. Kawaguchi Y, Nishiyama K, Miyagi K, Suzuki O, Ito Y, Nakamura $\mathrm{S}$. Patterns of failure associated with involved field radiotherapy in patients with clinical stage I thoracic esophageal cancer. Jpn J Clin Oncol. 2011;41:1007-1012.

7. Welsh J, Settle SH, Amini A, et al. Failure patterns in patients with esophageal cancer treated with definitive chemoradiation. Cancer. 2012; 118:2632-2640.

8. Zhao KL, Ma JB, Liu G, Wu KL, Shi XH, Jiang GL. Three-dimensional conformal radiation therapy for esophageal squamous cell carcinoma: is elective nodal irradiation necessary? Int J Radiat Oncol Biol Phys. 2010;76:446-451.

9. Zhang X, Li M, Meng X, et al. Involved-field irradiation in definitive chemoradiotherapy for locally advanced esophageal squamous cell carcinoma. Radiat Oncol. 2014;9:64.

10. Liu M, Zhao K, Chen Y, Jiang GL. Evaluation of the value of ENI in radiotherapy for cervical and upper thoracic esophageal cancer: a retrospective analysis. Radiat Oncol. 2014;9:232.

11. Edge SB, Byrd DR, Compton CC, Fritz AG, Greene FL, Tritti A, editors. AJCC Cancer Staging Manual. 7th ed. NewYork: Springer; 2010:103-104.

12. Therasse P, Arbuck SG, Eisenhauer EA, et al. New guidelines to evaluate the response to treatment in solid tumors. European Organization for Research and Treatment of Cancer, National Cancer Institute of the United States, National Cancer Institute of Canada. J Natl Cancer Inst. 2000;92:205-216.

13. Rice TW, Rusch VW, Ishwaran H, Blackstone EH; Worldwide Esophageal Cancer Collaboration. Cancer of the esophagus and esophagogastric junction: data-driven staging for the seventh edition of the American Joint Committee on Cancer/International Union Against Cancer Cancer Staging Manuals. Cancer. 2010;116:3763-3773.
14. Denham JW, Steigler A, Kilmurray J, et al. Relapse patterns after chemo-radiation for carcinoma of the oesophagus. Clin Oncol ( $R$ Coll Radiol). 2003;15:98-108.

15. Reid TD, Davies IL, Mason J, Roberts SA, Crosby TD, Lewis WG. Stage for stage comparison of recurrence patterns after definitive chemoradiotherapy or surgery for oesophageal carcinoma. Clin Oncol (R Coll Radiol). 2012;24:617-624.

16. Ishihara R, Yamamoto $\mathrm{S}$, Iishi $\mathrm{H}$, et al. Factors predictive of tumor recurrence and survival after initial complete response of esophageal squamous cell carcinoma to definitive chemoradiotherapy. Int J Radiat Oncol Biol Phys. 2010;76:123-129.

17. Yamashita H, Okuma K, Wakui R, Kobayashi-Shibata S, Ohtomo K, Nakagawa K. Details of recurrence sites after elective nodal irradiation (ENI) using 3D-conformal radiotherapy (3D-CRT) combined with chemotherapy for thoracic esophageal squamous cell carcinoma - a retrospective analysis. Radiother Oncol. 2011;98:255-260.

18. Onozawa M, Nihei K, Ishikura S, et al. Elective nodal irradiation (ENI) in definitive chemoradiotherapy (CRT) for squamous cell carcinoma of the thoracic esophagus. Radiother Oncol. 2009;92(2):266-269.

19. Kersting S, Konopke R, Dittert D, et al. Who profits from neoadjuvant radiochemotherapy for locally advanced esophageal carcinoma? J Gastroenterol Hepatol. 2009;24:886-895.

20. Stahl M, Wilke H, Stuschke M, et al. Clinical response to induction chemotherapy predicts local control and long-term survival in multimodal treatment of patients with locally advanced esophageal cancer. J Cancer Res Clin Oncol. 2005;131:67-72.

21. Zhong Z, Gu X, Zhang Z, et al. Recombinant human endostatin combined with definitive chemoradiotherapy as primary treatment for patients with unresectable but without systemic metastatic squamous cell carcinoma of the oesophagus. Br J Radiol. 2012;85:e1104-e1109.

22. Rosenberg JC, Franklin R, Steiger Z. Squamous cell carcinoma of the thoracic esophagus: an interdisciplinary approach. Curr Probl Cancer. 1981;5(11):1-52. Review.

23. Ishikura $\mathrm{S}$, Nihei $\mathrm{K}$, Ohtsu $\mathrm{A}$, et al. Long-term toxicity after definitive chemoradiotherapy for squamous cell carcinoma of the thoracic esophagus. J Clin Oncol. 2003;21:2697-2702.
OncoTargets and Therapy

\section{Publish your work in this journal}

OncoTargets and Therapy is an international, peer-reviewed, open access journal focusing on the pathological basis of all cancers, potential targets for therapy and treatment protocols employed to improve the management of cancer patients. The journal also focuses on the impact of management programs and new therapeutic agents and protocols on

\section{Dovepress}

patient perspectives such as quality of life, adherence and satisfaction. The manuscript management system is completely online and includes a very quick and fair peer-review system, which is all easy to use. Visit http://www.dovepress.com/testimonials.php to read real quotes from published authors. 